\title{
Automated Assessment of Quantitative REM Sleep without Atonia for Diagnosis of REM Sleep Behavior Disorder
}

\author{
Jeong Hun Yang', Sang Ho Choi², Mi Hyun Lee³ ${ }^{3}$ Seong Min $\mathrm{Oh}^{4}$, \\ Jae-Won Choi', Jee Eun Park ${ }^{6}$, Kwang Suk Park ${ }^{7}$, and Yu Jin Lee ${ }^{3}$ \\ 'Department of Psychiatry, Seoul National University Hospital, Seoul, Korea \\ ${ }^{2}$ Interdisciplinary Program in Bioengineering, Seoul National University, Seoul, Korea \\ ${ }^{3}$ Department of Psychiatry and Center for Sleep and Chronobiology, Seoul National University College of Medicine and \\ Seoul National University Hospital, Seoul, Korea \\ ${ }^{4}$ Department of Psychiatry, Dongguk University Ilsan Hospital, Goyang, Korea \\ ${ }^{5}$ Department of Neuropsychiatry, Eulji General Hospital, Eulji University School of Medicine, Seoul, Korea \\ ${ }^{6}$ Department of Psychiatry, Seoul National University College of Medicine, Seoul, Korea \\ ${ }^{7}$ Department of Biomedical Engineering, Seoul National University College of Medicine, Seoul; \\ Institute of Medical and Biological Engineering, Medical Research Center, Seoul National University, Seoul, Korea
}

\begin{abstract}
Objective: This study was performed to determine the diagnostic cutoff value of quantified tonic and phasic rapid eye movement (REM) sleep without atonia (RSWA) automatically calculated from chin and limb muscle electromyograms (EMG) for diagnosis of REM sleep behavior disorder (RBD). Methods: Nocturnal video polysomnographic data of 57 patients diagnosed with RBD and 29 age- and sex-matched controls were reviewed. Tonic activity was measured using submentalis EMG, and phasic activity was measured using submentalis and bilateral anterior tibialis EMG. The proportion of epochs with tonic and phasic activity during the entire REM sleep period was quantified using a self-developed automated algorithm. Results: The RBD group showed significantly more tonic activity compared with the control group $(28.87 \pm 36.92 \%$ vs. $12.94 \pm 31.69 \%$, respectively, $\mathrm{p}<0.001)$. The diagnostic cutoff value of quantified submentalis tonic RSWA for RBD showing the best optimal sensitivity and specificity was $0.99 \%$ [sensitivity, $77.2 \%$; specificity, $79.3 \%$, area under the receiver operating characteristic curve (AUC), 0.76]. Cutoffs of phasic RSWA were $47.53 \%$ when assessed in the submentalis only (sensitivity, $1.8 \%$; specificity, $100 \%$; AUC, 0.46 ), $0.10 \%$ in the anterior tibialis (sensitivity, 66.7\%; specificity, 55.2\%; AUC, 0.55), and $0.10 \%$ in both the submentalis and anterior tibialis (sensitivity, $70.2 \%$; specificity, 51.7\%; AUC, 0.53). Conclusion: This study provided evidence for the diagnosis of RBD using an automated method by assessing RSWA. Tonic activity in the submentalis muscle showed better sensitivity and specificity for diagnosis of RBD than did phasic activity.
\end{abstract}

Key Words: REM sleep behavior disorder; REM sleep without atonia; Automated algorithm

Received: June 26, 2020 Revised: August 11, 2020 Accepted: August 12, 2020

Corresponding author: Yu Jin Lee, MD, PhD, Department of Psychiatry and Center for Sleep and Chronobiology, Seoul National University College of Medicine and Seoul National University Hospital, 101 Daehak-ro, Jongno-gu, Seoul 03080, Korea.

Tel: 82-2-2072-2456, Fax: 82-2-744-7241, E-mail: ewpsyche@snu.ac.kr

(a) This is an Open Access article distributed under the terms of the Creative Commons Attribution Non-Commercial License (https://creativecommons.org/licenses/by$\mathrm{nc}$ 4.0) which permits unrestricted non-commercial use, distribution, and reproduction in any medium, provided the original work is properly cited.

\section{INTRODUCTION}

Rapid eye movement (REM) sleep behavior disorder (RBD) is a disorder characterized by the loss of muscle atonia that normally appears during REM sleep and the resulting body movements. RBD is associated with alpha-synucleinopathies, such as Parkinson's disease, Lewy body dementia, and multiple systemic atro- phy [1], and recent studies have shown that the degree of REM sleep without atonia (RSWA) predicts alpha-synucleionpathies $[2,3]$. Therefore, early detection of RBD is becoming increasingly important.

According to the American Academy of Sleep Medicine (AASM) diagnostic criteria, a diagnosis of RBD requires repeated behaviors during REM sleep, and tonic activity in the chin or phasic ac- 
tivity in the chin or limbs during polysomnography (PSG) must be observed [4]. However, as cutoff values for evaluating the presence or absence of tonic activity or phasic activity were not specified, the clinician's judgment is important for diagnosis. Fully automated methods to quantify RSWA and accurately diagnose RBD would greatly reduce the time and cost required for early diagnosis and treatment of the condition.

A number of studies have attempted to quantify RSWA in PSG to diagnose RBD. In 1992, Lapierre and Montplaisir [5] quantified RSWA by manual scoring in sleep PSG. In 2008, Ferri et al. [6] calculated the REM atonia index using an automated method. Most subsequent studies regarding the diagnosis of RBD have used automated algorithms following Ferri's method. However, unlike visual scoring, the REM atonia index algorithm evaluates baseline electromyogram (EMG) activity without conforming to the typical tonic/phasic system and a 30-s epoch and 3-s mini epoch system used by AASM as diagnostic criteria, making it difficult to compare with human-rated diagnosis according to AASM. Jeppesen et al. [7] used a semi-automated algorithm to quantify and compare tonic and phasic activities, but their method requires manual removal of all of the epochs with artifacts prior to analysis to ensure high diagnostic accuracy [area under the receiver operating characteristic (ROC) curve (AUC) $=0.940$ (tonic), 0.975 (phasic), and 0.978 (any)]. There have been no previous studies of the accuracy of tonic or phasic RSWA calculated using a fully automated method for the diagnosis of RBD. According to recent reports, tonic and phasic RSWA have different clinical implications in RBD. It was suggested that phasic activity was related to the violent expressions of dream-enacting behaviors whereas tonic activity was more closely related to disease progression to synuceinopathies [3]. It is expected that different aspects of RBD could be analyzed by calculating and quantifying tonic and phasic respectively.

Moreover, most previous studies that used automated methods measured RSWA in RBD only in the chin muscles. Previous studies using visual scoring reported that the sensitivity and specificity for the chin muscles were significantly lower than those for the limb muscles in predicting phasic motor activity during sleep [8-10]. In those studies, the diagnostic accuracy of phasic activity increased significantly when limb muscles, especially the flexor digitorum superficialis and extensor digitorum brevis, were evaluated along with the chin muscles. Therefore, in the AASM guidelines, when evaluating phasic activity, clinicians are instructed to evaluate chin EMG as well as limb EMG. To establish a more accurate method for automated quantification of RSWA and diagnosis of RBD, it is necessary to compare the diagnostic accuracies of tonic and phasic RSWA measurements in the chin and limb according to the AASM diagnostic criteria.

In this study, we attempted to quantify tonic RSWA and phasic RSWA according to the AASM diagnostic criteria using an automated method, and to obtain the optimal RSWA value that accurately predicts RBD. Tonic RSWA was measured in the chin muscle only, and phasic RSWA was measured in both the chin and limb muscles. RSWA values derived using these methods were compared to determine which method predicts RBD most accurately.

\section{METHODS}

\section{Participants}

We retrospectively reviewed the medical records of patients $>50$ years old who underwent nocturnal video PSG (v-PSG) at the Sleep and Chronobiology Center of Seoul National University Hospital between January 1, 2016, and May 31, 2019. The exclusion criteria were 1) current or historical major psychiatric illnesses (schizophrenia, schizoaffective disorder, bipolar disorder, and major depressive disorder) or neurodegenerative diseases, including $\alpha$-synucleinopathies; 2) current signs or symptoms of parkinsonism; 3) any current or previous serious medical illness, including ischemic heart disease, arrhythmia, or diabetes mellitus; 4) apnea-hypopnea index $>15$; and 5) periodic limb movement index $>15$.

Fifty-seven patients ( 36 males, 21 females) who underwent v-PSG during the study period were diagnosed with RBD at the sleep clinic of the psychiatric department according to clinical evaluation and v-PSG based on International Classification of Sleep Disorders-3 [11] and who did not meet the exclusion criteria were enrolled in the study. The control group consisted of participants who underwent v-PSG during the same period but who did not have a diagnosis or clinical suspicion of sleep disorders including RBD, obstructive sleep apnea (OSA), or restless leg syndrome (RLS) and who did not meet the exclusion criteria. We selected 29 ageand sex-matched healthy controls (18 males and 11 females) from among those who met the criteria for the healthy control group.

This retrospective study was approved by the Institutional Review Board of Seoul National University Hospital (approval no. $\mathrm{H}-1809-080-97)$. All procedures were performed in accordance with the ethical standards of the research committee and were implemented in accordance with the 1964 Declaration of Helsinki and subsequent amendments.

v-PSG

At the Center for Sleep and Chronobiology of Seoul National University Hospital, data from overnight v-PSG (Profusion3; Compumedics, Charlotte, NC, USA) consisting of electroencephalograms (electrodes at F3, F4, C3, C4, O1, and $\mathrm{O} 2$, using $\mathrm{A} 1$ and A2 as reference sites), bilateral electrooculograms, a single-lead electrocardiogram (lead II), submentalis and bilateral anterior tibialis EMG, airflow measurement via a nasal pressure transducer and oronasal thermal sensor, a respiratory inductance plethysmography band to monitor the movements of the chest and abdomen, and a finger pulse oximeter were analyzed. Polysomnographic data were scored by experienced technicians and physicians in accordance with the AASM recommendations [4]. The parameters apnea-hypopnea index, periodic limb movement index, time in bed, total sleep time, sleep efficiency, wake after sleep onset, sleep latency, REM latency, and percentage of sleep in each stage were 
calculated and recorded.

\section{RSWA analysis}

In principle, the scoring method in this study followed the standard of RBD scoring method based on AASM as possible in a fully automated way. First, EMG signals from the submentalis and bilateral anterior tibialis muscle were digitally band-pass filtered at $10-100 \mathrm{~Hz}$ to exclude artifacts. Then, RSWA was quantified as proposed by Lapierre and Montplaisir [5] by dividing the entire PSG into epochs and mini-epochs and calculating the rates of tonic epochs and phasic mini-epochs among all epochs and mini-epochs. Here, we defined epochs as $30 \mathrm{~s}$ and mini-epochs as $3 \mathrm{~s}$ based on the AASM scoring manual $[4,10]$. According to the AASM manual, tonic activity was measured using chin EMG, whereas phasic activity was measured using chin or limb EMG. In previous studies, phasic activity was measured 1) only in the mentalis or submentalis muscle $[5,12], 2)$ in the bilateral anterior tibialis muscle and the mentalis or submentalis muscle [13], or 3 ) in other limb muscles, such as the flexor digitorum superficialis, biceps brachii, or extensor digitorum brevis muscles, in addition to the mentalis or submentalis and bilateral anterior tibialis muscles $[9,10,14]$. No submentalis or bilateral anterior tibialis EMG data were available for 12 of 57 participants with RBD and 24 of 28 control subjects in our study; therefore, we assessed only the submentalis and bilateral anterior tibialis to detect phasic activity.

In the present study, when an epoch of REM sleep had an EMG amplitude $>10 \mu \mathrm{V}$ in the submentalis EMG channel during at least $50 \%$ of the duration of the epoch, it was defined as an epoch with tonic activity. On the other hand, when determining phasic activity, a 30-s epoch of REM sleep was divided into 10 sequential 3 -s mini-epochs. When at least five (50\%) of the mini-epochs contained bursts of transient muscle activity with a burst duration $>3 \mathrm{~s}$ and amplitude $>10 \mu \mathrm{V}$ in the submentalis or anterior tibialis EMG channel, the epoch was defined as having phasic activity. The cutoff amplitude of tonic and phasic activity was set to $10 \mu \mathrm{V}$ (rather than $2 \times$ and $4 \times$ the stage $\mathrm{R}$ atonia activity, as defined by the AASM) because the background EMG activity of the samples could not always be determined easily [10]. The RSWA of tonic activity was defined as the rate of tonic epochs of all the epochs of REM sleep, and the RSWA of phasic activity was defined as the rate of phasic mini-epochs of all the mini-epochs of REM sleep.

\section{Statistical analysis}

To summarize the demographic, clinical, and polysomnographic variables, descriptive statistics were used. The Mann-Whitney U-test was used to compare continuous variables and Fisher's exact test to compare categorical variables to assess the demographic, clinical, and polysomnographic differences between groups.

We used ROC curves to display the true positives and false positives, regarding the diagnosis in medical records according to International Classification of Sleep Disorders-3 as the gold standard. We measured the AUC, which indicates the accuracy of automatically calculated RSWA for diagnosis of RBD. The opti- mal cutoff value of RSWA that maximizes the sensitivity and specificity of the diagnosis was calculated using Youden's index [15]. Statistical analyses were performed using the Statistical Package for the Social Sciences version 23.0 (IBM Corp., Armonk, NY, USA). In all analyses, $\mathrm{p}<0.05$ was taken to indicate statistical significance.

\section{RESULTS}

\section{Demographic and clinical characteristics}

The demographic and polysomnographic characteristics of the RBD and control groups are shown in Table 1. There were no significant differences in sociodemographic or clinical characteristics between the two groups. As the RBD and control groups were matched for both age and sex, there were no statistically significant differences in age ( $67.11 \pm 6.56$ years vs. $67.18 \pm 6.08$ years, respectively, $\mathrm{p}=0.87$ ) or sex [ 21 of $57(36.8 \%)$ females vs. 11 of 29 $(37.9 \%)$ females, $\mathrm{p}=0.92]$ between the two groups. There were also no significant differences between the two groups in terms of polysomnographic variables, including the percentage duration of each sleep stage, except for the percentage of stage N2, which was lower in the RBD group than the control group $(52.02 \pm 12.48 \%$ vs. $56.57 \pm 7.79 \%$, respectively, $\mathrm{p}=0.046$ ).

\section{Comparisons of phasic and tonic RSWA}

Comparison of RSWA between the RBD and control groups indicated a statistically significant difference only in tonic RSWA (Table 2). Tonic RSWA was significantly higher in the RBD group than the control group $(28.87 \pm 36.92 \%$ vs. $12.94 \pm 31.69 \%$, respectively, $\mathrm{p}<0.001)$. Phasic RSWA was not significantly different between the two groups when measured in the submentalis only,

Table 1. Comparisons of demographic and polysomnographic characteristics between the RBD patients and control groups

\begin{tabular}{lccc}
\multicolumn{1}{c}{ Variable } & $\begin{array}{c}\mathrm{RBD} \\
(\mathrm{n}=57)\end{array}$ & $\begin{array}{c}\text { Control } \\
(\mathrm{n}=29)\end{array}$ & p-value \\
Age (yr) & $67.11 \pm 6.56$ & $67.18 \pm 6.08$ & 0.87 \\
Sex, female & $21(36.8)$ & $11(37.9)$ & 0.92 \\
TIB (min) & $481.99 \pm 31.30$ & $482.16 \pm 25.90$ & 0.75 \\
TST (min) & $381.61 \pm 49.71$ & $379.40 \pm 59.31$ & 0.96 \\
Sleep efficiency (\%) & $79.57 \pm 11.28$ & $78.69 \pm 11.53$ & 0.68 \\
WASO (min) & $85.57 \pm 57.13$ & $90.67 \pm 53.02$ & 0.54 \\
Sleep latency (min) & $19.21 \pm 23.44$ & $11.60 \pm 10.75$ & 0.06 \\
REM latency (min) & $117.82 \pm 64.03$ & $102.48 \pm 53.84$ & 0.31 \\
Stage N1 (\%) & $23.51 \pm 13.14$ & $20.39 \pm 9.13$ & 0.51 \\
Stage N2 (\%) & $52.02 \pm 12.48$ & $56.57 \pm 7.79$ & 0.046 \\
Stage N3 (\%) & $5.13 \pm 7.45$ & $3.38 \pm 5.32$ & 0.33 \\
Stage R (\%) & $19.35 \pm 7.54$ & $19.66 \pm 6.40$ & 0.83 \\
AHI & $6.61 \pm 4.06$ & $5.89 \pm 3.89$ & 0.31 \\
PLMI & $25.73 \pm 36.55$ & $12.65 \pm 18.99$ & 0.08 \\
\hline
\end{tabular}

Data are presented as numbers (\%) or means \pm standard deviations. RBD: rapid eye movement sleep behavior disorder, TIB: time in bed, TST: total sleep time, WASO: wake after sleep onset, REM: rapid eye movement, AHI: apnea-hypopnea index, PLMI: periodic limb movement index 
Table 2. Comparisons of tonic and phasic RSWA between the RBD patient and control groups

$\begin{array}{cccc}\text { Variable } & \mathrm{RBD} & \begin{array}{c}\text { Control } \\ (\mathrm{n}=57)\end{array} & \text { p-value }\end{array}$

Tonic RSWA (\%)

Submentalis

$28.87 \pm 36.92$

$12.94 \pm 31.69$

$<0.001$

Phasic RSWA (\%)

$\begin{array}{llll}\text { Submentalis } & 1.56 \pm 10.81 & 0.96 \pm 2.90 & 0.35 \\ \text { Anterior tibialis } & 2.40 \pm 4.72 & 5.66 \pm 11.88 & 0.45 \\ \begin{array}{l}\text { Submentalis+ } \\ \text { anterior tibialis }\end{array} & 3.96 \pm 11.47 & 6.45 \pm 12.07 & 0.61\end{array}$

Data are presented as means \pm standard deviations. RBD: rapid eye movement sleep behavior disorder, RSWA: rapid eye movement sleep without atonia the anterior tibialis only, or both muscles.

Construction of ROC curves and calculation of the AUC

ROC curves were constructed for tonic and phasic RSWA to determine the cutoff value with the highest sensitivity and specificity to distinguish RBD patients from controls (Figure 1). For tonic RSWA measured in the submentalis muscle, the optimal cutoff value was $0.99 \%$ (sensitivity, $77.2 \%$; specificity, $79.3 \%$; AUC, 0.76). Meanwhile, the optimal cutoff value of phasic RSWA was $47.53 \%$ when assessed in the submentalis muscle only (sensitivity, $1.8 \%$; specificity, $100 \%$; AUC, 0.46 ), $0.10 \%$ in the anterior tibialis muscle (sensitivity, $66.7 \%$; specificity, $55.2 \%$; AUC, 0.55 ), and $0.10 \%$ in both the submentalis and anterior tibialis muscles (sensitivity, 70.2\%; specificity, 51.7\%; AUC, 0.53) (Figure 1).
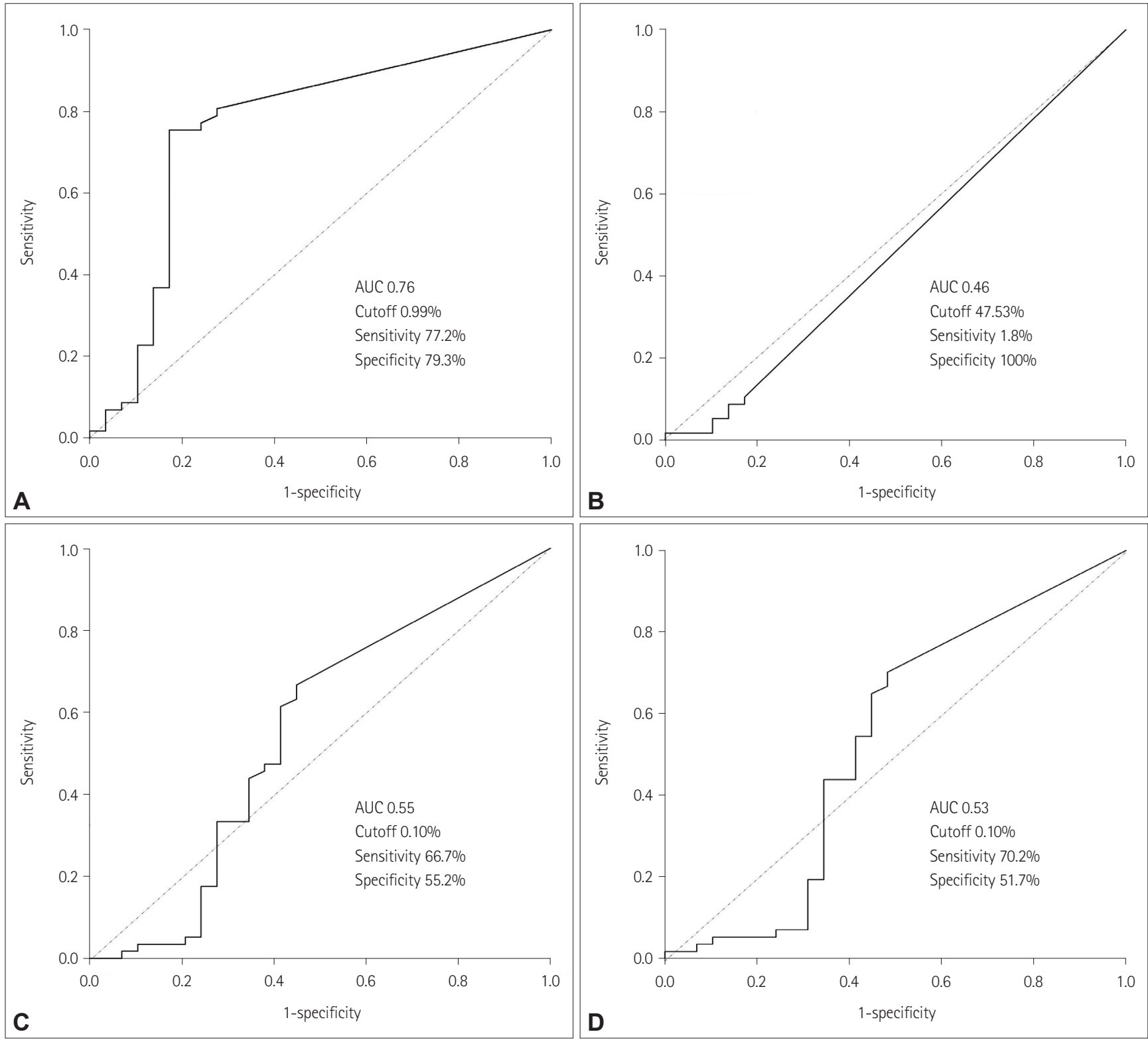

Figure 1. ROC curves evaluating the ability of RSWA to diagnose RBD. (A) Tonic RSWA in the submentalis. (B) Phasic RSWA in the submentalis. (C) Phasic RSWA in the anterior tibialis. (D) Phasic RSWA in both the submentalis and anterior tibialis. ROC: receiver operating characteristic, AUC: area under the ROC curve, RSWA: rapid eye movement sleep without atonia, RBD: rapid eye movement sleep behavior disorder. 


\section{DISCUSSION}

The present study was performed to assess the diagnostic accuracies of tonic and phasic RSWA calculated using a fully automated method, with the diagnosis by clinicians according to the conventional visual scoring method used as the gold standard. This is the first study to compare the accuracy of RWSA for detecting RBD by calculating both tonic and phasic RSWA using a fully automated method. It is also the first study to evaluate RSWA in chin muscles as well as limb muscles using a fully automated method in RBD. However, the diagnostic accuracy of RSWA evaluated using AUC values was lower in this study than in previous studies.

Unlike Jeppesen's semi-automated method [7], our study used a fully automated algorithm, and manual screening and exclusion of artifacts were not performed before RSWA analysis. However, this method could not fully correct artifacts, including sleep-apnea related events and dream enactment behaviors. Artifacts may have remained in our study after filtering at $10-100 \mathrm{~Hz}$, making it difficult to obtain a constant baseline stage $\mathrm{R}$ atonia EMG amplitude. We defined tonic and phasic RSWA as an EMG amplitude $>10 \mu \mathrm{V}$ instead of using the conventional criteria of $2 \times$ and $4 \times$ the amplitude of stage $\mathrm{R}$ atonia for tonic and phasic activities, respectively [4], which may have been responsible for the reduced specificity of the test.

In addition, in a significant number of controls, tonic or phasic RSWA was detected at a level capable of diagnosing RBD despite the absence of the disease, which led to a further decline in accuracy. In fact, RSWA in controls was observed in previous studies evaluating the diagnostic accuracy of tonic and phasic RSWA measured in the chin muscle [16,17]. Even in normal REM sleep, brief phasic activity may appear in the form of twitching [18], which may have contributed to a reduction in the diagnostic specificity of phasic RSWA in the present study. Moreover, in older subjects, even without a history or symptoms of RBD, the tendency of REM atonia to decrease with age as a result of degenerative processes was reported $[19,20]$. The age of the participants in the present study (67.11 years in the RBD group and 67.18 years in the control group) was the highest among studies reported to date quantifying RSWA using automated methods. It is possible that the diagnostic value of the test declined as RSWA in the control increased as a result of aging. In addition, as RSWA showed a relatively continuous distribution among the participants in both groups, it is possible that the control group included subjects who had not yet been diagnosed or shown manifestations of RBD. Moreover, the RBD cases in the present study consisted of idiopathic RBD without neurodegenerative diseases, such as Parkinson's disease, and thus the symptoms were likely to be relatively mild, which may have led to even smaller differences in RSWA from the control group than in previous studies. Considering the risk of falls during sleep or future transition to alpha-synucleinopathies, the need for more active screening and diagnosis of RBD is suggested.

In this study, tonic RSWA showed higher diagnostic accuracy than did phasic RSWA. In previous studies using visual scoring in patients with Parkinson's disease, tonic activity showed superior diagnostic accuracy compared with phasic activity [21]. In other studies, tonic rather than phasic RSWA was associated with more specific findings of RBD, such as higher neurodegeneration rates, and the authors suggested that degeneration of the sublaterodorsal nucleus, which is a characteristic of RBD transition to alphasynucleinopathy, may be correlated with tonic RSWA [22,23]. The reason for the difference in the diagnostic accuracy between tonic and phasic RSWA in our study is not clear, but considering that $\mathrm{RBD}$ is considered an early manifestation of alpha-synucleinopathy [24], tonic rather than phasic RSWA may be related more directly to the core clinical features of RBD. However, this interpretation must be evaluated carefully, as other studies showed no difference in diagnostic accuracy between tonic and phasic RSWA $[7,17,25]$.

Tonic RSWA showed relatively good sensitivity and specificity for the diagnosis of RBD, but the cutoff value was lower than those in previous reports using the manual method (0.99-30\%) $[16,17,20,21]$ and similar to that in a previous study using a semiautomated method (2.2\% for tonic) [7]. The automated method appeared to detect small changes in RSWA more sensitively than did the visual method, so the disease could be diagnosed even when using a lower cutoff. On the other hand, the sensitivity of phasic RSWA measured in the chin was only $1.8 \%$, which is not acceptable as a sole diagnostic tool, but the diagnostic accuracy was improved by also evaluating phasic activity in the limb muscles. It is expected that phasic RSWA will show better accuracy in future studies using a combination of upper limb muscles that have been reported to show superior accuracy, such as the extensor digitorum brevis or flexor digitorum superficialis [8-10,21]. Moreover, AASM requires a duration of $0.1-5.0 \mathrm{~s}$ to diagnose phasic activity. In contrast, this study applied a stricter standard by requiring phasic RSWA to last for the entire mini-epoch (3 s), which would have further reduced the sensitivity of phasic RSWA.

Meanwhile, the diagnostic accuracy of the quantified RSWA in this study was even lower than that of previous studies using the fully automated method. This is thought to be because the previous studies minimized the effects of artifacts using the REM atonia index, which might be useful in that the effect of artifacts could be reduced without manual inspection, but could not calculate tonic and phasic activities separately. In our study, we calculated tonic and phasic activity respectively, which could extract quantified RSWA with higher clinical value but might have lead to the increase in the effect of artifacts and decrease in the diagnostic accuracy.

This study had some limitations. First, 57 patients with RBD was an insufficient number to establish a diagnostic method for the disease. However, it is still a relatively large sample size compared to previous studies that attempted to derive diagnostic cutoff through quantified scoring of RBD. This is the highest number except for the Ferris study in 2012, which analyzed 74 RBD patients [19]. Second, the possibility of selection bias cannot be excluded because all participants were tested at a single sleep clinic. 
Third, it is difficult to estimate the diagnostic value in young patients by limiting the target to participants over 50 years old. Finally, although we performed filtering to exclude artifacts, we did not perform manual inspection of artifacts, thereby reducing the diagnostic accuracy. Due to the limitations mentioned above, the diagnostic accuracy of the AUC curve presented in current study might have decreased. However, this study, which quantified RSWA through a fully automated method with a relatively large sample size, has clinical and exploratory significance in proposing an alternative automated method for scoring of RBD to the conventional manual scoring method.

In summary, we quantified tonic and phasic RSWA using fully automated techniques in RBD patients and normal controls, thereby evaluating RSWA accuracy for diagnosing RBD. Tonic RSWA showed greater diagnostic accuracy than that of phasic RSWA, which showed better accuracy when measured in limb than chin muscles. To improve the diagnostic accuracy, further prospective studies in more population of various age groups with effective filtering of artifacts are required.

\section{Acknowledgments}

None

\section{Conflicts of Interest}

The authors have no potential conflicts of interest to disclose.

\section{Author Contributions}

Conceptualization: Jeong Hun Yang, Yu Jin Lee. Data curation: Jeong Hun Yang, Sang Ho Choi, Mi Hyun Lee, Seong Min Oh. Formal analysis: Jeong Hun Yang, Sang Ho Choi. Investigation: Jeong Hun Yang, Jae-Won Choi. Methodology: Jeong Hun Yang, Kwang Suk Park. Project administration: Jeong Hun Yang, Jee Eun Park. Resources: Jeong Hun Yang, Yu Jin Lee. Software: Jeong Hun Yang, Sang Ho Choi. Supervision: Yu Jin Lee. Validation: Jeong Hun Yang, Mi Hyun Lee. Visualization: Jeong Hun Yang, Seong Min Oh. Writing_original draft: Jeong Hun Yang, Jae-Won Choi. Writing_review \& editing: Jee Eun Park, Yu Jin Lee.

\section{ORCID iDs}

Yu Jin Lee (D)

https://orcid.org/0000-0001-5195-2579

Jeong Hun Yang (D)

https://orcid.org/0000-0002-1286-264X

\section{REFERENCES}

1. Högl B, Stefani A. REM sleep behavior disorder (RBD): update on diagnosis and treatment. Somnologie (Berl) 2017;21(Suppl 1):1-8.

2. Nepozitek J, Dostalova S, Dusek P, Kemlink D, Prihodova I, Ibarburu Lorenzo Y Losada V, et al. Simultaneous tonic and phasic REM sleep without atonia best predicts early phenoconversion to neurodegenerative disease in idiopathic REM sleep behavior disorder. Sleep 2019;42:zsz132.

3. McCarter SJ, Sandness DJ, McCarter AR, Feemster JC, Teigen LN, Timm PC, et al. REM sleep muscle activity in idiopathic REM sleep behavior dis- order predicts phenoconversion. Neurology 2019;93:e1171-e1179.

4. Berri RB, Brooks R, Garnaldo CE, Harding SM, Lloyd RM, Marcus CL, et al. The AASM manual for the scoring of sleep and associated events: rules, terminology and technical specifications. Ver. 2.5. Darien, IL: American Academy of Sleep Medicine, 2018.

5. Lapierre O, Montplaisir J. Polysomnographic features of REM sleep behavior disorder: development of a scoring method. Neurology 1992;42:13711374.

6. Ferri R, Manconi M, Plazzi G, Bruni O, Vandi S, Montagna P, et al. A quantitative statistical analysis of the submentalis muscle EMG amplitude during sleep in normal controls and patients with REM sleep behavior disorder. J Sleep Res 2008;17:89-100.

7. Jeppesen J, Otto M, Frederiksen Y, Hansen AK, Fedorova TD, Knudsen K, et al. Observations on muscle activity in REM sleep behavior disorder assessed with a semi-automated scoring algorithm. Clin Neurophysiol 2018;129:541547.

8. Iranzo A, Frauscher B, Santos H, Gschliesser V, Ratti L, Falkenstetter T, et al. Usefulness of the SINBAR electromyographic montage to detect the motor and vocal manifestations occurring in REM sleep behavior disorder. Sleep Med 2011;12:284-288.

9. Frauscher B, Iranzo A, Högl B, Casanova-Molla J, Salamero M, Gschliesser $\mathrm{V}$, et al. Quantification of electromyographic activity during REM sleep in multiple muscles in REM sleep behavior disorder. Sleep 2008;31:724-731.

10. Frauscher B, Iranzo A, Gaig C, Gschliesser V, Guaita M, Raffelseder V, et al. Normative EMG values during REM sleep for the diagnosis of REM sleep behavior disorder. Sleep 2012;35:835-847.

11. American Academy of Sleep Medicine. International classification of sleep disorders. 3rd ed. Darien, IL: American Academy of Sleep Medicine, 2014.

12. Dauvilliers Y, Rompré S, Gagnon JF, Vendette M, Petit D, Montplaisir J. REM sleep characteristics in narcolepsy and REM sleep behavior disorder. Sleep 2007;30:844-849.

13. Iranzo A, Muñoz E, Santamaria J, Vilaseca I, Milà M, Tolosa E. REM sleep behavior disorder and vocal cord paralysis in Machado-Joseph disease. Mov Disord 2003;18:1179-1183.

14. Iranzo A, Ratti PL, Casanova-Molla J, Serradell M, Vilaseca I, Santamaria J. Excessive muscle activity increases over time in idiopathic REM sleep behavior disorder. Sleep 2009;32:1149-1153.

15. Youden WJ. Index for rating diagnostic tests. Cancer 1950;3:32-35.

16. Montplaisir J, Gagnon JF, Fantini ML, Postuma RB, Dauvilliers Y, Desautels A, et al. Polysomnographic diagnosis of idiopathic REM sleep behavior disorder. Mov Disord 2010;25:2044-2051.

17. Lee SA, Kim CS, Cho CU, Kim B, Lee GH. Quantitative EMG criteria for diagnosing idiopathic REM sleep behavior disorder. Sleep Breath 2015;19:685691.

18. Schupp M, Hanning CD. Physiology of sleep. BJA CEPD Rev 2003;3:69-74.

19. Ferri R, Bruni O, Fulda S, Zucconi M, Plazzi G. A quantitative analysis of the submentalis muscle electromyographic amplitude during rapid eye movement sleep across the lifespan. J Sleep Res 2012;21:257-263.

20. Feemster JC, Jung Y, Timm PC, Westerland SM, Gossard TR, Teigen LN, et al. Normative and isolated rapid eye movement sleep without atonia in adults without REM sleep behavior disorder. Sleep 2019;42:zsz124.

21. Figorilli M, Ferri R, Zibetti M, Beudin P, Puligheddu M, Lopiano L, et al. Comparison between automatic and visual scorings of REM sleep without atonia for the diagnosis of REM sleep behavior disorder in Parkinson disease. Sleep 2017;40:Zsw060.

22. Liu Y, Zhang J, Lam SP, Yu MWM, Li SX, Zhou J, et al. Electromyography activity level in rapid eye movement sleep predicts neurodegenerative diseases in idiopathic rapid eye movement sleep behavior disorder: a 5-year longitudinal study. Sleep Med 2019;56:128-134.

23. Postuma RB, Gagnon JF, Rompré S, Montplaisir JY. Severity of REM atonia loss in idiopathic REM sleep behavior disorder predicts Parkinson disease. Neurology 2010;74:239-244.

24. Schenck CH. Rapid eye movement sleep behavior disorder: current knowledge and future directions. Sleep Med 2013;14:699-702.

25. Consens FB, Chervin RD, Koeppe RA, Little R, Liu S, Junck L, et al. Validation of a polysomnographic score for REM sleep behavior disorder. Sleep 2005;28:993-997. 